\title{
Performance Analysis of Scientific and Engineering Applications Using MPInside and TAU
}

\author{
Subhash Saini ${ }^{1}$, Piyush Mehrotra ${ }^{1},{\text { Kenichi } \text { Taylor }^{2} \text {, Sameer Shende }}^{3}$, Rupak Biswas ${ }^{1}$ \\ ${ }^{1}$ NASA Advanced Supercomputing ${ }^{2}$ Silicon Graphics International (SGI) \\ NASA Ames Research Center \\ Moffett Field, CA 94035 USA \\ 46600 Landing Pkwy \\ \{subhash.saini, piyush.mehrotra, \\ Fremont, CA 94538 USA \\ rupak.biswas\}@nasa.gov \\ kenichi@sgi.com \\ ${ }^{3}$ ParaTools, Inc. \\ 2836 Kincaid Street \\ Eugene, OR 97405 USA \\ sameer@paratools.com
}

\begin{abstract}
In this paper, we present performance analysis of two NASA applications using performance tools like Tuning and Analysis Utilities (TAU) and SGI MPInside. MITgemUV and OVERFLOW are two production-quality applications used extensively by scientists and engineers at NASA. MITgcmUV is a global ocean simulation model, developed by the Estimating the Circulation and Climate of the Ocean (ECCO) Consortium, for solving the fluid equations of motion using the hydrostatic approximation. OVERFLOW is a general-purpose Navier-Stokes solver for computational fluid dynamics (CFD) problems. Using these tools, we analyze the MPI functions (MPI_Sendrecv, MPI_Bcast, MPI_Reduce, MPI_Allreduce, MPI_Barrier, etc.) with respect to message size of each rank, time consumed by each function, and how ranks communicate. MPI communication is further analyzed by studying the performance of MPI functions used in these two applications as a function of message size and number of cores. Finally, we present the compute time, communication time, and I/O time as a function of the number of cores.
\end{abstract}

\section{INTRODUCTION}

Developing or porting codes on new computing architectures to achieve good performance is a challenging and daunting task for application scientists and engineers. Performance of most of the real-world applications is less than $10 \%$ of the peak performance on these computing systems. Low performance is due to a number of challenges facing the high-performance scientific community, including increasing levels of parallelism (threads, multi- and many-cores, nodes), deeper and more complex memory hierarchies (register, multiple levels of cache, on node NUMA memory, disk, network), and hybrid hardware (processors and GPGPUs). In many cases, factors such as runtime variation due to system noise, traditional computer benchmarking is not sufficient to understand the performance of large-scale applications. In such cases, simple inspection of the profile (the timing breakdown) is not adequate to analyze performance of particularly MPI applications. One needs to know what is happening "inside" both the application and the MPI library and along with the interaction of the two.
The present study uses two performance tools (SGI's MPInside and TAU from University of Oregon) to profile two production-quality applications (OVERFLOW-2 and MITgcmUV, hereafter OVERFLOW-2 will be referred as OVERFLOW). This study also uses the low-level MPI function benchmarks to measure their performance as a function of message size. The study was carried out on an SGI Altix ICE 8200EX cluster, Pleiades, located at NASA Ames Research Center. Pleiades consists of two subclusters: one part based uses the Xeon 5472 Harpertown processor [1-2] (hereafter called "Pleiades-HT"), and the second uses Xeon 5570 Nehalem processor, the first server implementation of a new 64-bit micro-architecture (henceforth called "Pleiades-NH") [3-4]. All the nodes employ the Linux operating system and SGI MPT library and are connected in a hypercube topology using InfiniBand [5-6].

In this paper we have conducted the performance profiling of OVERFLOW and MITgcmUV using the two performance tools, MPInside and TAU, on Pleiades-HT and Pleiades-NH. We have also evaluated and compared the performance of MPI functions as a function of message size on Pleiades-HT and Pleiades-NH.

The remainder of this paper is organized as follows: Section 2 describes the two performance tools, SGI's MPInside and TAU from University of Oregon, used in the study. Section 3 gives the overview of the applications and MPI function benchmarks. Section 4 presents and analyzes results from running these benchmarks and applications on the two clusters. Section 5 contains a summary and conclusions of the study.

\section{Performance Tools Used}

Based on an initial survey and looking into pros and cons of each performance tool, we decided to use two tools, MPInside and TAU, to conduct in-depth performance analysis of two real-world applications used extensively by scientists and engineers at NASA [7-22]. MPInside is a profiling and diagnostic tool developed by SGI to analyze and predict the performance of MPI applications [15]. 
Tuning and Analysis Utilities (TAU) developed by University of Oregon, and supported by ParaTools, Inc., is a portable profiling and tracing toolkit for performance analysis of parallel programs [14].

\section{Applications and Benchmarks}

\subsection{Science and Engineering Applications}

For this study, we used two production applications, taken from NASA's workload. OVERFLOW, developed at NASA's Langley Research Center, is a general-purpose Navier-Stokes solver for CFD problems [23]. MITgcmUV, developed by the Estimating the Circulation and Climate of the Ocean (ECCO) Consortium, is a global ocean simulation model for solving the fluid equations of motion using the hydrostatic approximation [24].

\subsection{Intel MPI Benchmarks (IMB)}

The performance of real-world applications that use MPI as the programming model depends significantly on the MPI library and the performance of various point-to-point and collective message exchange operations supported by the MPI implementations. Intel MPI Benchmarks (IMB), (formerly, the Pallas MPI Benchmarks) is a commonly used benchmark suite to evaluate and compare the performance of different MPI implementations [25].

The MPI standard defines several collective operations, which can be broadly classified into three major categories based on the message exchange pattern: OnetoAll, AlltoOne, and Alltoall. We have evaluated the performance of MPI_Bcast, MPI_Reduce, MPI_Alltoall, and MPI_Allreduce collective operations on both clusters.

\section{Results}

In this section, we present the results of our study.

\subsection{Scientific and Engineering Applications}

\subsubsection{MITgcmUV}

Figure 1 shows the sustained performance of MITgcmUV using TAU and MPInside.

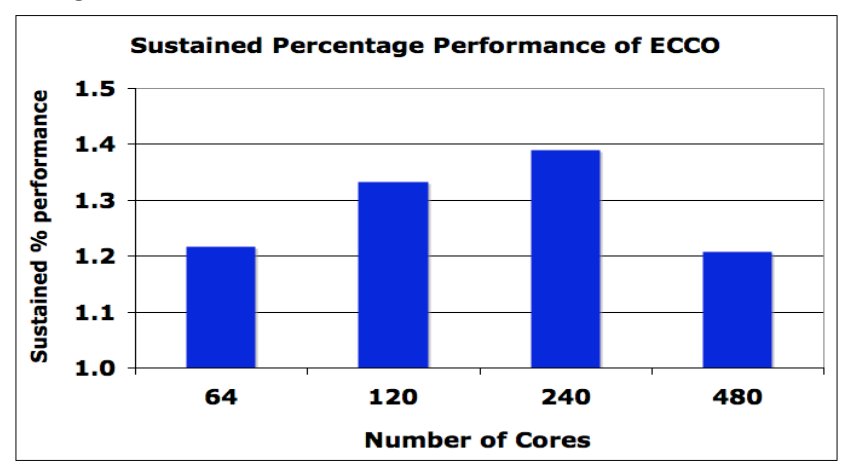

Figure 1: Sustained performance of MITgcmUV on Pleiades-HT

Sustained performance of MITgcmUV is about $1.2-1.4 \%$ of the peak, which is relatively low, as most of the applications have sustained performance around 3-8\% of peak. We have not looked into the cause of this low performance from processor and memory subsystem perspective here but have only investigated the role of various MPI functions for the application.

In Figure 2, we show the percentage of time for total, compute, communication, and I/O times on Pleiades-HT and Pleiades-NH. As expected, percentage of compute time decreases and communication time increases for increasing numbers of cores for both systems. Percentage contribution of $\mathrm{I} / \mathrm{O}$ time increases for large number of cores. On 64 cores of Pleiades-NH compute is $93 \%$, communication $3.5 \%$, $\mathrm{I} / \mathrm{O}$ $3.5 \%$. Corresponding numbers for 480 cores are: compute $59.1 \%$, communication $23.4 \%$, and $\mathrm{I} / \mathrm{O} 17.5 \%$.

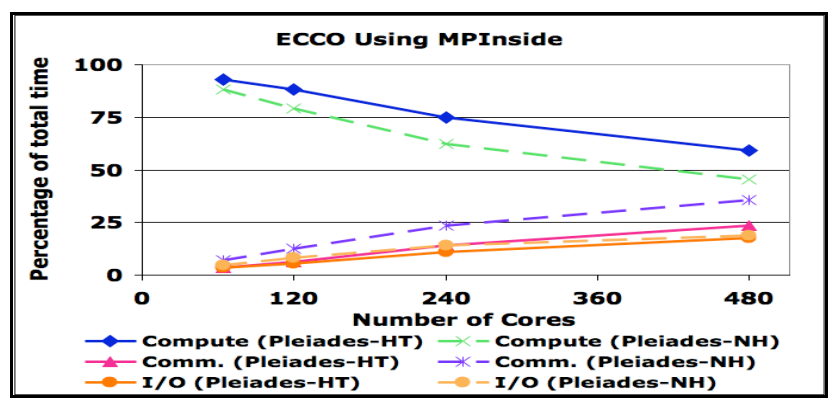

Figure 2: Time percentage of MITgcmUV using MPInside on two systems.

Figure 3 shows the read, write, and (read+write) times for MITgcmUV on two systems. Read time is almost the same on both systems; however, write time on Pleiades-HT is much higher than on Pleiades- $\mathrm{NH}$ - it writes $8 \mathrm{~GB}$ of data. This is due to the fact that Pleiades-NH has three times more memory than Pleiades-HT so one is measuring writes to buffer cache in memory. On the other hand, on PleiadesHT one is measuring "write time" to disk because there is not enough memory to hold all 8 GBs of output data.

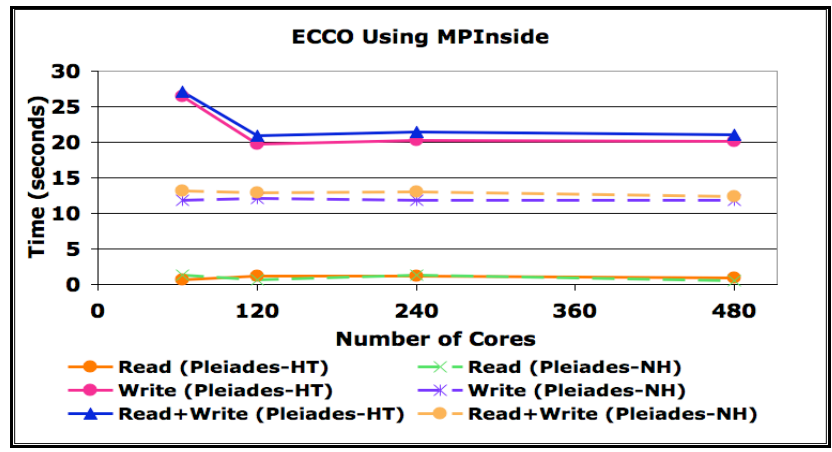

Figure 3: I/O times for MITgcmUV using MPInside on two systems.

In Figure 4, we plot the write bandwidth on the two systems. Write bandwidth on Pleiades-NH is about $55 \%$ higher than on Pleiades-HT. As mentioned in the previous paragraph, this is due to the fact that there is three times more memory per core in Pleiades-NH than Pleiades-HTwrite is done using the memory buffer as opposed to disk write in Pleiades-HT. 


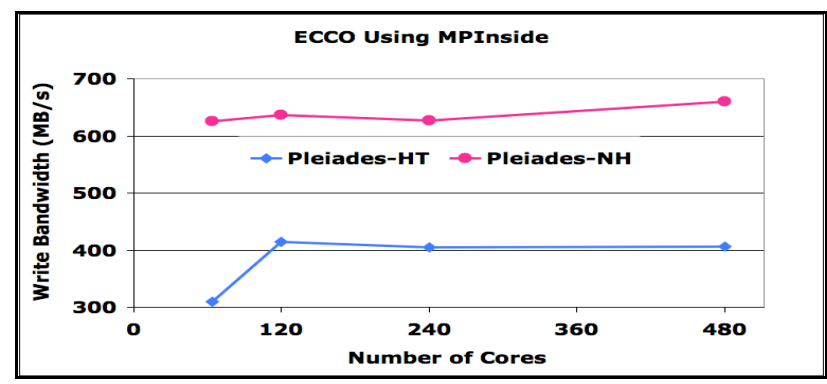

Figure 4: Write bandwidth of MITgcmUV on two systems.

In Figure 5, we plot the percentage of time spent in each of the MPI functions in MITgcmUV. Percentage of communication time spent is 60,30, and 5\% in MPI_Recv, MPI_Allreduce, and MPI_Waitall, respectively. Only 5\% of the time is spent in MPI_Send, MPI_Isend, MPI_Bcast, and MPI_Barrier.

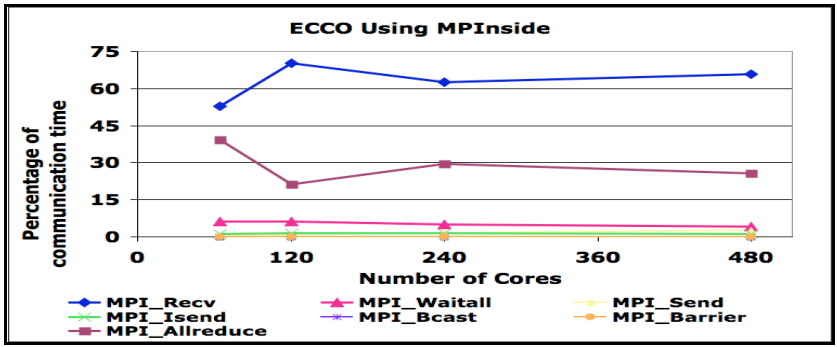

Figure 5: Percentage of time spent in MPI functions for MITgcmUV.

In Figure 6, we plot the minimum, average, and maximum message size of MPI_Recv in MITgcmUV using MPInside. The average message size varies from 3-9 KB.

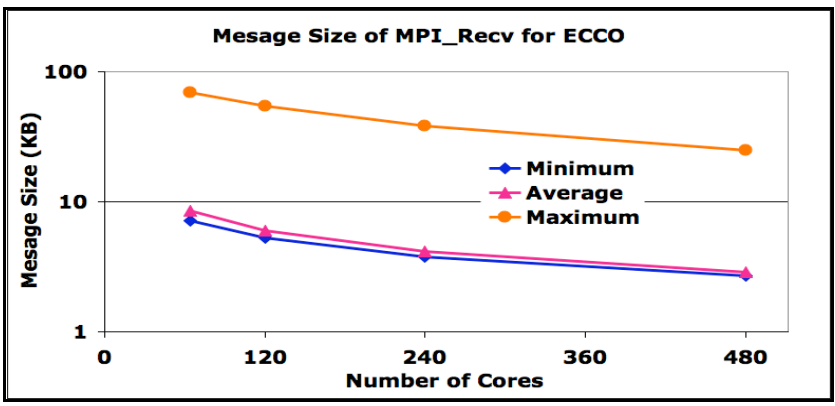

Figure 6: Message size of MPI_Recv in MITgcmUV using MPInside.

With both the tools the message size in MPI_Allreduce is 8 bytes for cores ranging from 60 to 480 . Since data size is only 8 bytes, MPI Allreduce is network latency-bound in MITgcmUV. A message size of $225 \mathrm{~KB}$ is broadcast to all cores. Message sizes for all MPI functions in MITgcmUV including MPI_Recv, MPI_Allreduce, and MPI_Bcast were the same, as measured by TAU and MPInside.

\subsubsection{OVERFLOW}

In this subsection, we present results for OVERFLOW using the performance tools MPInside and TAU. Only the results obtained using MPInside are shown, as they are same as those obtained by using TAU.
Figure 7 shows the sustained performance of OVERFLOW. Sustained performance is about $2.5 \%$ of peak. Performance of OVERFLOW is slightly better than MITgcmUV. We notice that even for 16 cores (2 nodes), performance is low. We did not investigate the cause of this low sustained performance but believe it is related to processor and memory subsystem.

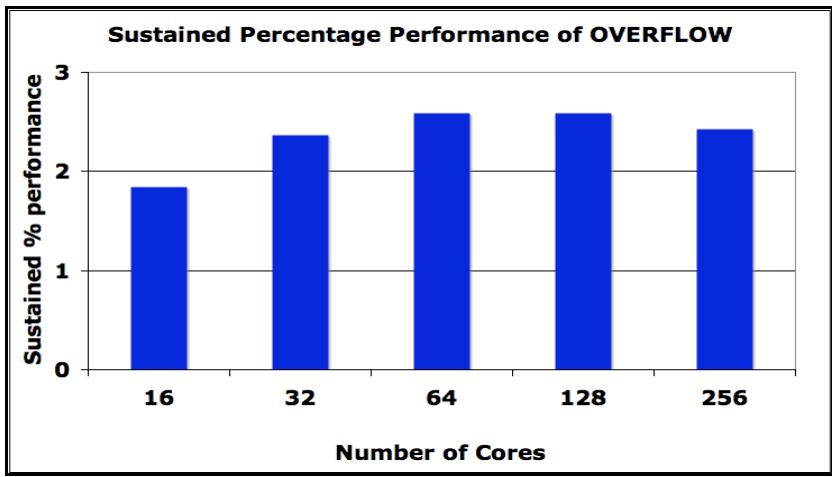

Figure 7: Sustained performance of OVERFOW.

Figure 8 shows the percentage of computation, communication, I/O, and total time on both systems. On both systems, percentage of computation time decreases as the number of cores increase from 32 to 128 and then increases for 256 cores. In addition, percentage of communication time increases as the number of cores increases from 32 to 128 , and then decreases for 256 cores. For 256 cores on Pleiades-HT: computation 62\%, communication $25 \%$, and $\mathrm{I} / \mathrm{O} 13 \%$; Pleiades-NH: computation $52 \%$, communication $33 \%$, and $\mathrm{I} / \mathrm{O} 15 \%$.

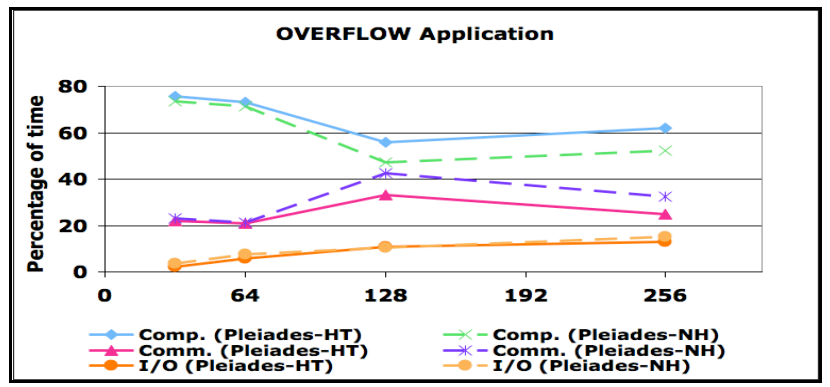

Figure 8: Percentage of computation, communication, I/O, and total time in OVERFLOW using MPInside.

Figure 9 shows the I/O time for OVERFLOW on the two

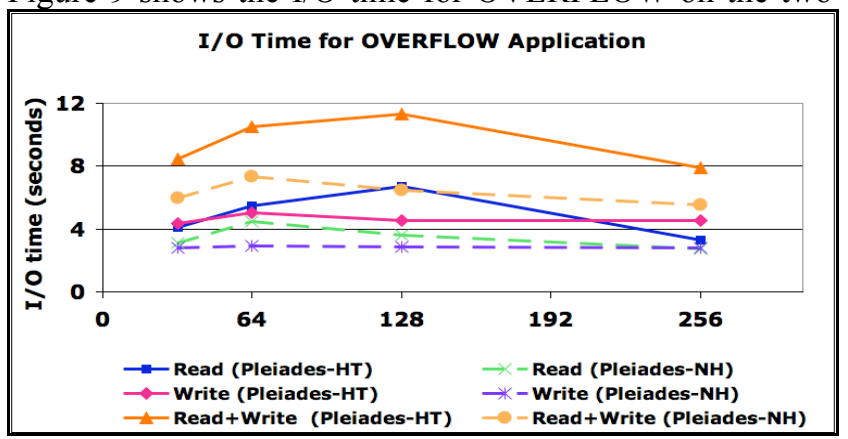

Figure 9: I/O time in OVERFLOW for Pleiades-HT and Pleiades-NH. 
systems. I/O times are better on Pleiades-NH than on Pleiades-HT. Performance of (read+write) is better on Pleiades-NH than Pleiades-HT by a factor of 1.4 for all core counts except at 128 cores where it is a factor of 1.7.

Figure 10 shows the read and write bandwidth in OVERFLOW for the two systems. Size of input data file read is $1.6 \mathrm{~GB}$, and size of the solution file written is 2 GB. Both read and write bandwidths are higher on Pleiades-NH than on Pleiades-HT. The reason for this is that memory per node is three times higher on PleiadesNH than on Pleiades-HT (24 vs. $8 \mathrm{~GB}$ ), so size of memory buffers is higher in the former. Performance of the write bandwidth in OVERFLOW is almost the same as in MITgcmUV, although data written is four times larger in MITgcmUV (2 vs. $8 \mathrm{~GB})$.

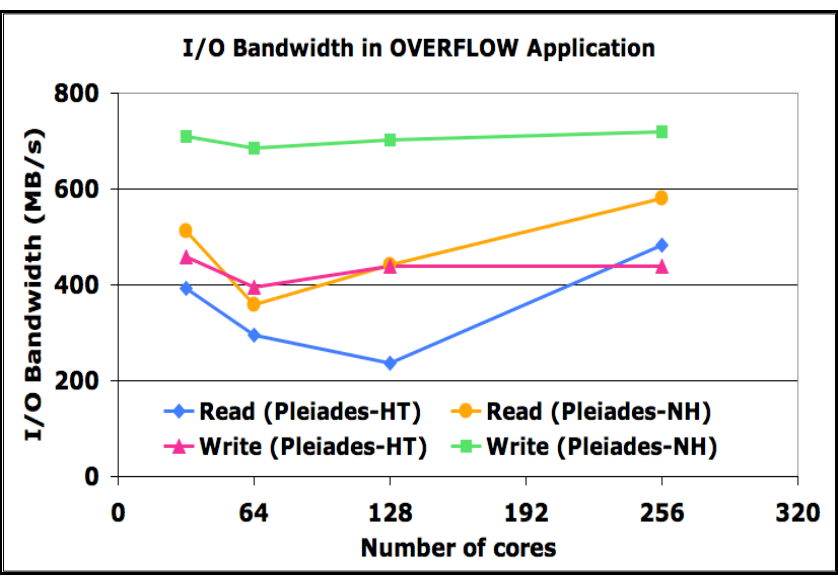

Figure 10: Read and write bandwidth in OVERFLOW for two systems.

In Figure 11, we show times for the top five MPI functions. Most of the time is consumed by the two functions MPI_Waitall and MPI_Gatherv, followed by MPI_Recv and MPI_Send and the lowest time by MPI_Bacst. For 128-256 cores, time for MPI_Waitall and MPI_GatherV decreases, whereas time for MPI_Recv, $M P I$ Send, and MPI_Bcast remains almost constant.

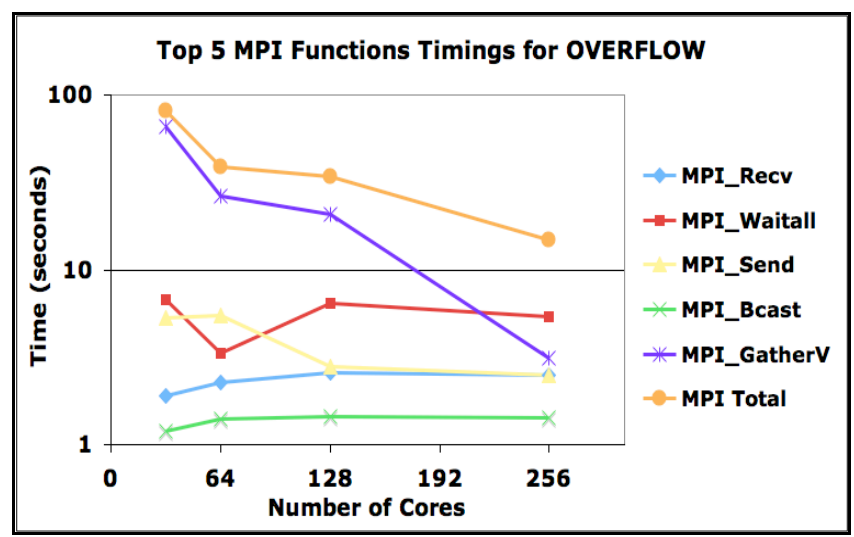

Figure 11: Timings for the top 5 MPI functions in OVERFLOW.

Figure 12 shows percentage time for the top $5 \mathrm{MPI}$ functions. Percentage of time taken by $M P I$ Recv and MPI_Send increases as the number of cores increases. Up to 64 cores, percentage of time taken by MPI_Send is more than MPI_Recv and then it becomes the same for 128 and 256 cores. For higher numbers of cores, percentage time consumed by all MPI functions increases, except for MPI GatherV. At 256 cores, percentage of time consumed by $\bar{M} P I \_$Waitall is the highest. The function MPI_Waitall waits for all communications to complete. At $25 \overline{6}$ cores, percentage of time contributions are MPI_Waitall 36\%, MPI_GatherV 21\%, MPI_Recv 17\%, MPI_Send 17\%, and MPI_Bcast $9 \%$.

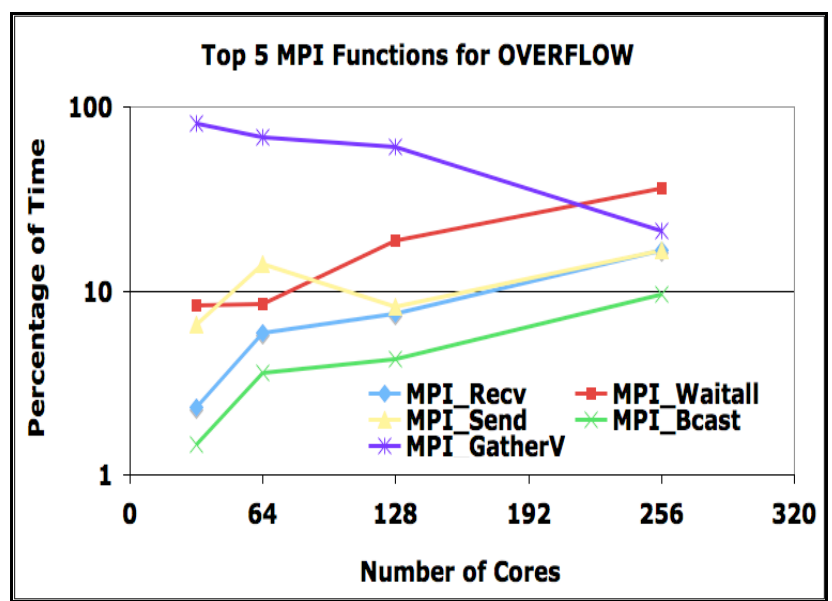

Figure 12: Percentage time for the top 5 MPI functions in OVERFLOW using MPInside.

Figure 13 shows the minimum, average, and maximum message size of MPI_Send in the OVERFLOW application. Message size decreases as the number of cores increases. The average message size for MPI_Send is 348, 129, 80, and $54 \mathrm{~KB}$ for 16, 128, 256, and 512 cores, respectively.

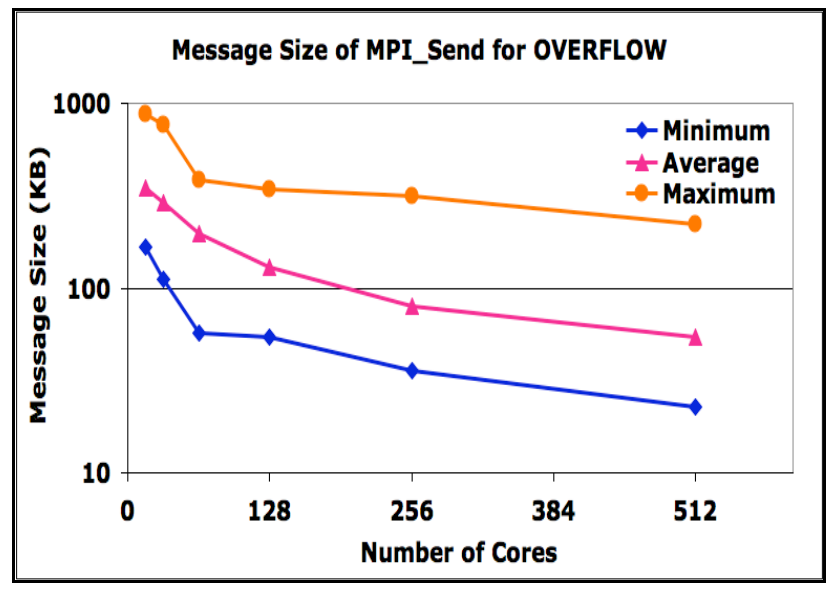

Figure 13: Message size for the MPI_Send function in OVERFLOW using MPInside.

Figure 14 shows the minimum, average, and maximum message size for MPI_Recv. For all three cases, size first increases up to $32 / 64$ cores, and then decreases up to 512 cores. Average message size for MPI_Recv is 53, 104, 144, and $219 \mathrm{~KB}$ for $16,128,256$, and 512 cores respectively. 


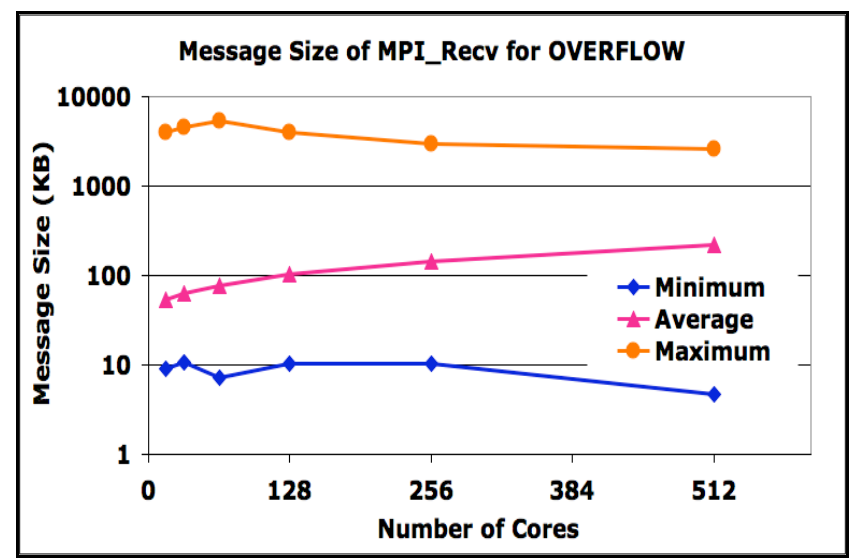

Figure 14: Message size for the MPI Recv function in OVERFLOW using MPInside.

Figure 15 shows the message size for MPI_Bcast. Message size for MPI_Bcast is $1.29 \mathrm{MB}$ in the OVERFLOW application from 16 to 512 cores.

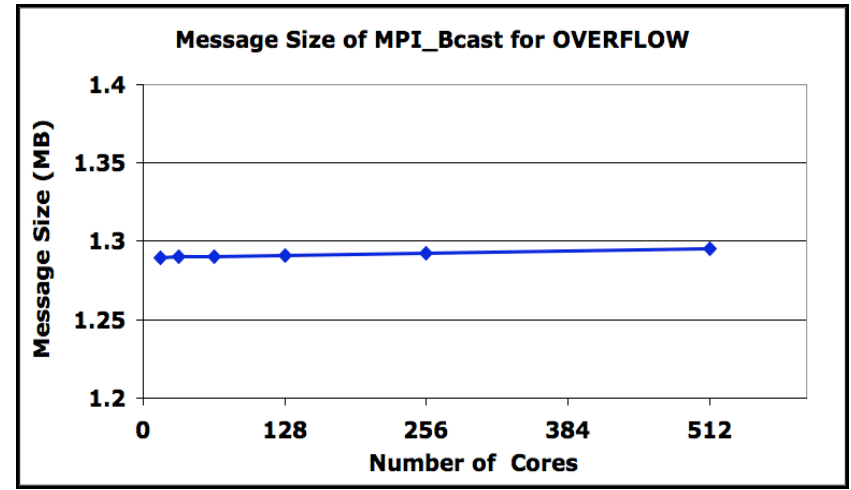

Figure 15: Message size for the MPI_Bcast function in OVERFOW using MPInside.

Figure 16 shows the minimum, average, and maximum message size for MPI_Gatherv. Average size of the message gathered by MPI_Gatherv is 270 bytes for 16 to 512 cores. Since the size of the message is very small, performance of MPI_Gatherv depends on network latency and not on network bandwidth.

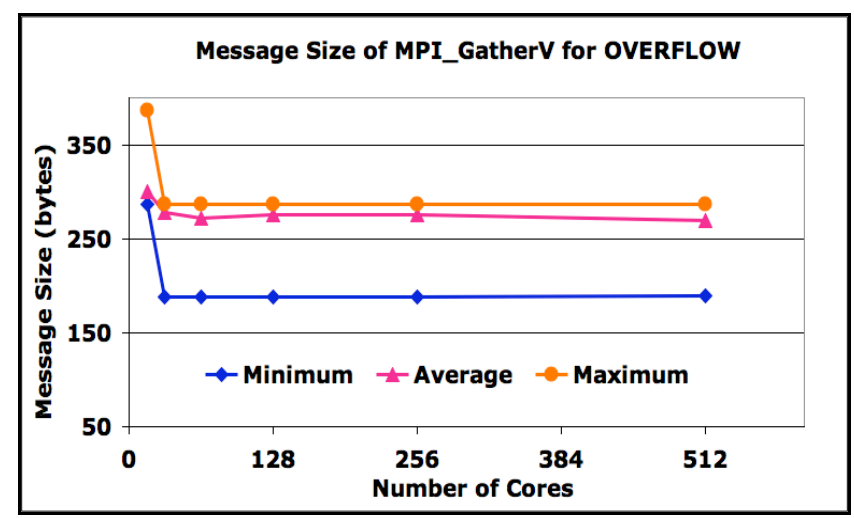

Figure 16: Message size for the MPI_Gatherv function in OVERFLOW using MPInside.

\subsection{Intel MPI Benchmarks (IMB)}

In this section, we describe the performance of various MPI functions relevant to the two applications (MITgcmUV and OVERFLOW) used in this paper.

\subsubsection{MPI_Sendrecv\& MPI_Exchange}

In Figure 17, we plot the performance of the MPI_Sendrecv and MPI_Exchange benchmarks for small messages on both systems. This plot provides insights into the relationship between the message exchange pattern, point-to-point message exchange algorithms, and overall performance. On both systems, performance of the MPI_Sendrecv benchmark is better than MPI_Exchange. In the MPI_Exchange benchmark, each process exchanges messages with both its left and right neighbors simultaneously, whereas in the MPI_Sendrecv benchmark, each process receives from its left neighbor and sends to its right neighbor at any instant. Since the MPI_Sendrecv benchmark involves a lesser volume of messages exchanged in comparison with MPI_Exchange, it is natural to expect better throughput. We see a change in slope for both benchmarks on the two systems around a message size of $1 \mathrm{~KB}$, which is due to a change of algorithm.

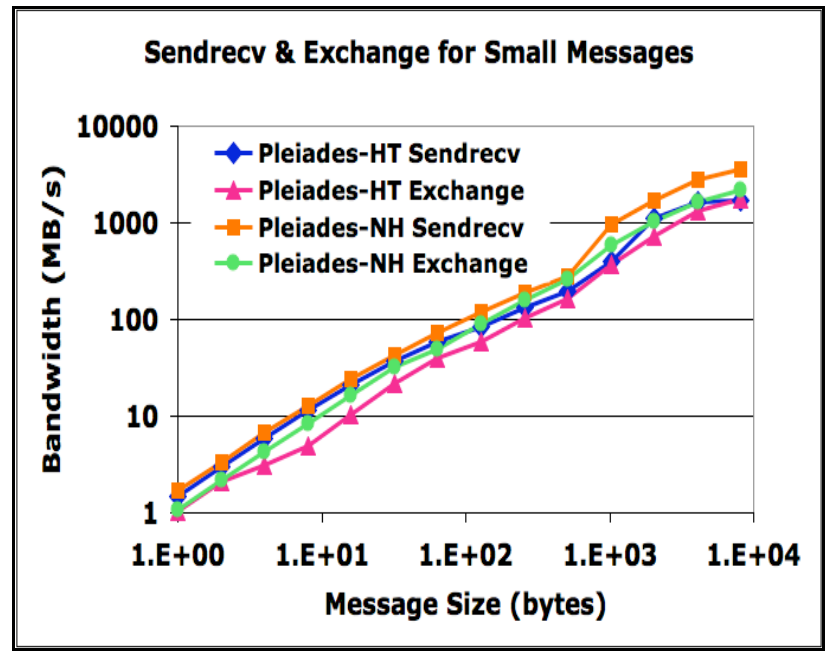

Figure 17: Performance of the MPI_Sendrecv and MPI_Exchange functions on two systems for small messages.

In Figure 18, we plot the performance of the MPI_Sendrecv and MPI_Exchange benchmarks for large messages on both systems. We see a peak bandwidth with a $16 \mathrm{~KB}$ message $(3.6 \mathrm{~GB} / \mathrm{s}$ for Pleiades-NH vs. $2.6 \mathrm{~GB} / \mathrm{s}$ for Pleiades-HT), which falls drastically for larger messages and stabilizes at $2.3 \mathrm{~GB} / \mathrm{s}$ for Pleiades-NH and 2.9 GB/s for Pleiades-HT. We believe this could be due to cache effects as large message intra-node exchanges usually involve making a copy from the user buffer to the shared-memory buffers. As size of the data in the user buffer grows, we may not be able to fit it in the cache, leading to cache misses. 


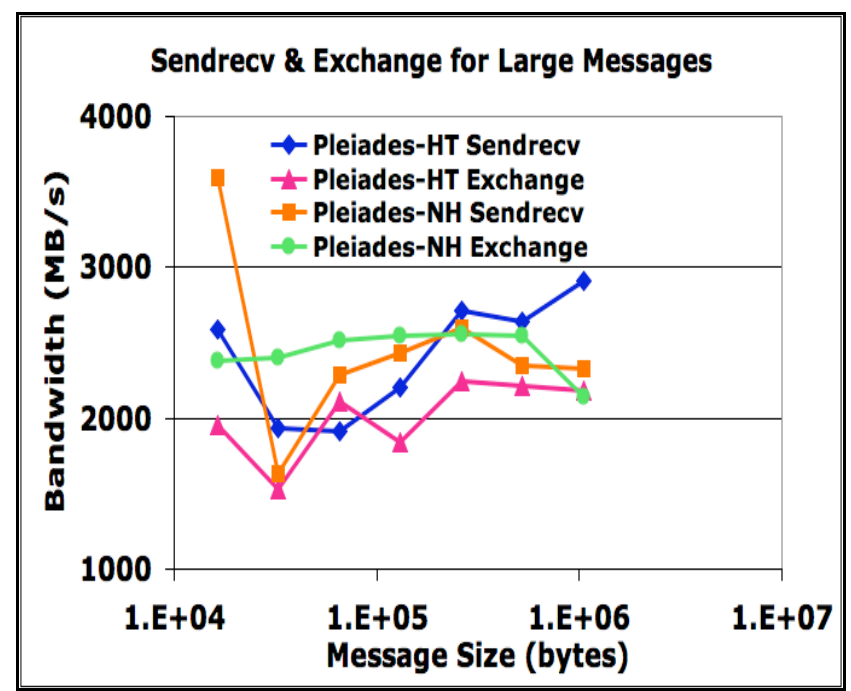

Figure 18: Performance of the MPI Sendrecv and MPI Exchange functions on two systems for large messages.

Figure 19 shows the bandwidth of the MPI Sendrecv benchmark for a message size of $262 \mathrm{~KB}$, which is the average size used in MITgcmUV for the cores ranging from 2 to 512. Bandwidth within a node $(8$ cores) is higher on Pleiades-NH than on Pleiades-HT as the former uses faster intra-node communication via QPI. Beyond 8 cores (a node), the bandwidth on both systems is almost same except at 512 cores where Pleiades-NH has higher bandwidth.

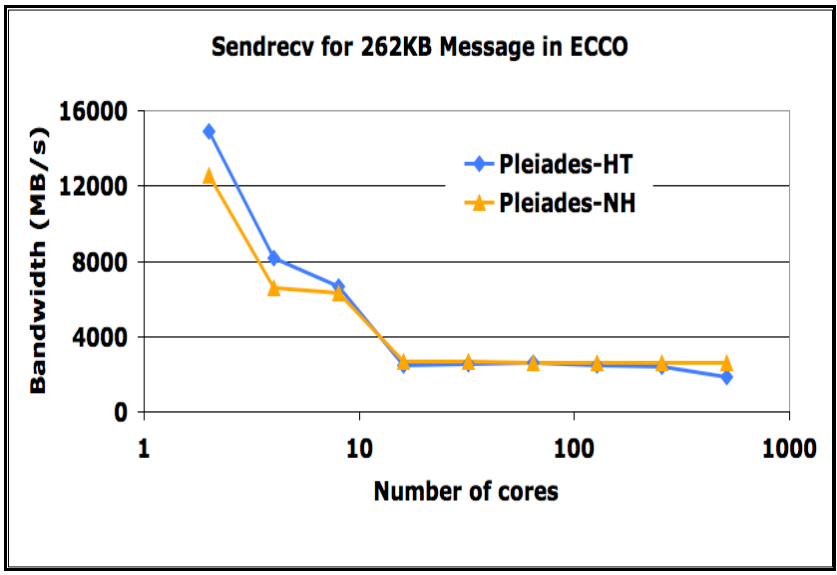

Figure 19: Performance of the MPI_Sendrecv function on two systems for a message size of $262 \mathrm{~KB}$.

\subsubsection{MPI_Bcast}

Figure 20 shows the performance of MPI_Bcast for small messages on the two systems. Up to a $1 \mathrm{~KB}$ message size, performance on both systems is almost the same. However, beyond that we notice there is a drastic change of slope on both systems due to transition of algorithms used in its implementation. In addition, performance is better on Pleiades-NH than on Pleiades-HT.

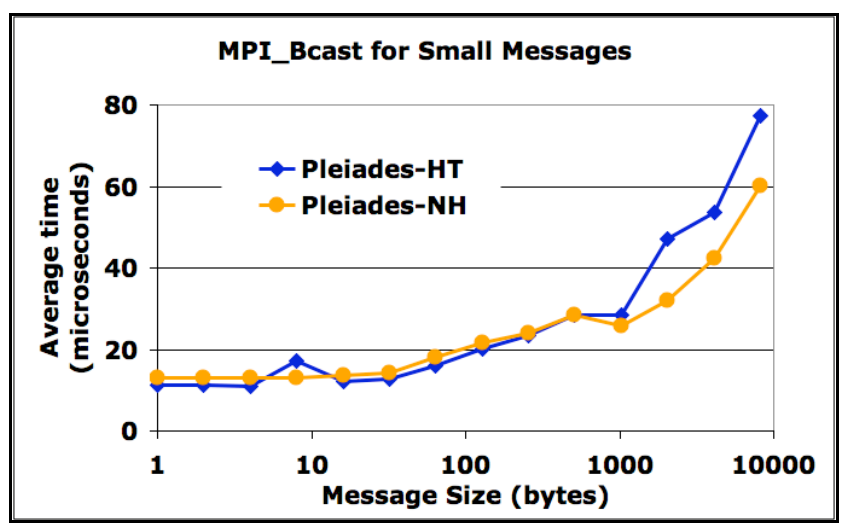

Figure 20: Performance of MPI Bcast on two systems for small messages.

Figure 21 shows the performance of MPI_Bcast for large messages on the two systems. Performance difference between the two systems is small for 16 to $64 \mathrm{~KB}$, and then the performance gap increases as the message size increases. Timings are (a) $64 \mathrm{~KB}: 289$ vs. $481 \mu \mathrm{s}$, and (b) 1 MB: 5,398 vs. $8,038 \mu$ s on two systems.

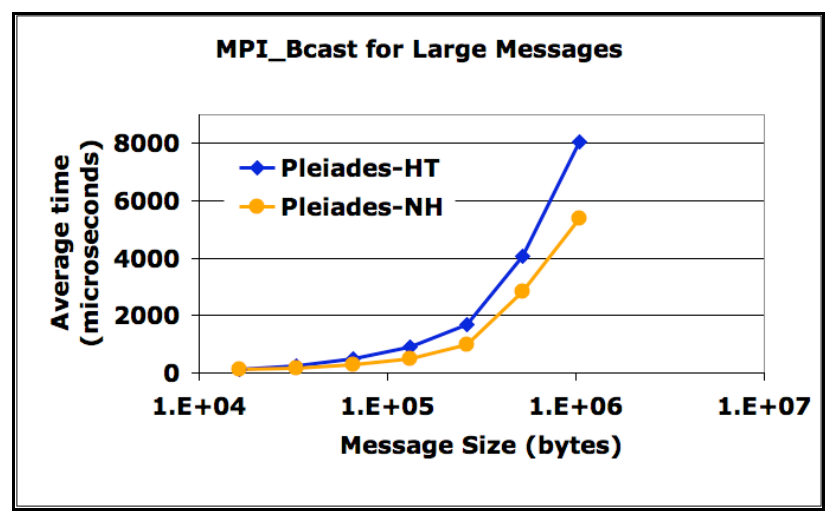

Figure 21: Performance of MPI_Bcast on two systems for large messages. Figure 22 shows the performance of MPI Bcast for a 1 MB message size used in OVERFLOW. We see that performance on Pleiades-NH is higher than Pleiades-HT for both intra- and inter-node communication.

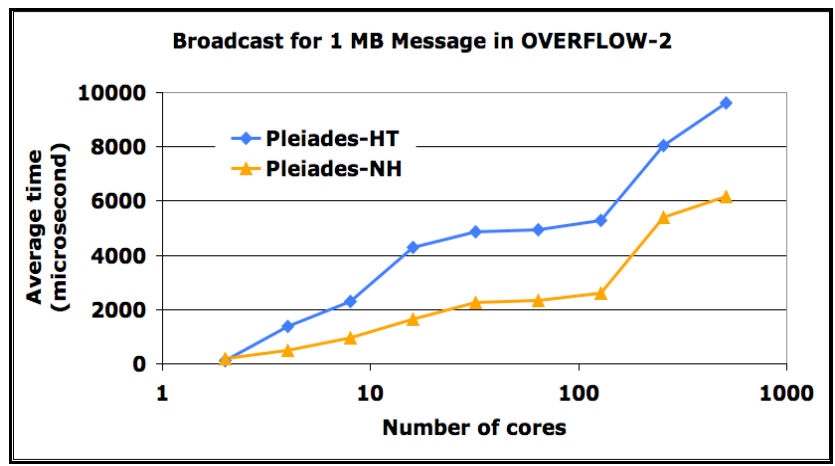

Figure 22: Performance of MPI_Bcast on two systems for a $1 \mathrm{MB}$ message. 


\subsubsection{MPI_Allreduce}

In Figure 23, we plot average time for the MPI_Allreduce benchmark for small messages for both systems. Up to 64 bytes, performance is higher on Pleiades-HT and then from 128 bytes to $1 \mathrm{~KB}$, performance is the same. From $2 \mathrm{~KB}$ onwards, the performance gap continues to widen and at 8 $\mathrm{KB}$, it is $40 \%$ higher (151 vs. $211 \mu \mathrm{s})$.

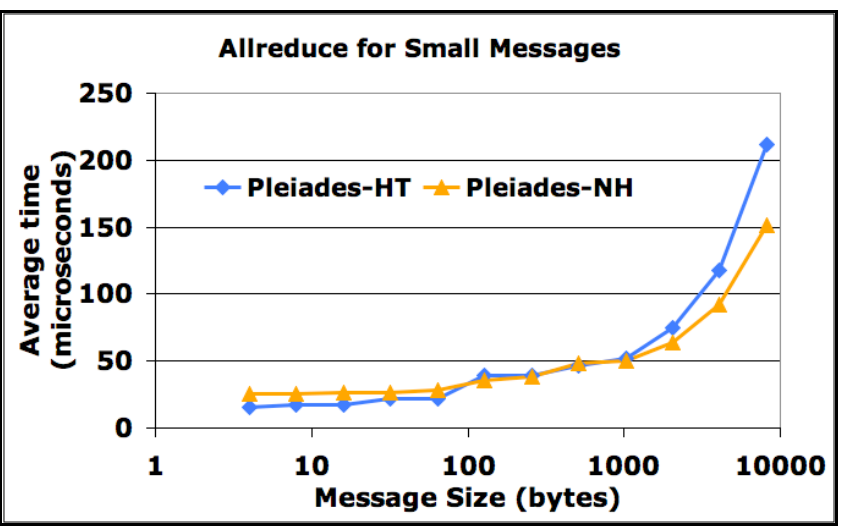

Figure 23: Performance of MPI_Allreduce on two systems for small messages.

In Figure 24, we plot the average time for the MPI_Allreduce benchmark for large messages for both systems. Throughout all cores, performance on Pleiades$\mathrm{NH}$ is higher than on Pleiades-HT, and the performance gap increases as the number of cores increases. At $16 \mathrm{~KB}$, times are 261 and $392 \mu \mathrm{s}$, and at $1 \mathrm{MB}$, they are 7,958 and $10,897 \mu$ s for Pleiades-NH and Pleiades-HT, respectively.

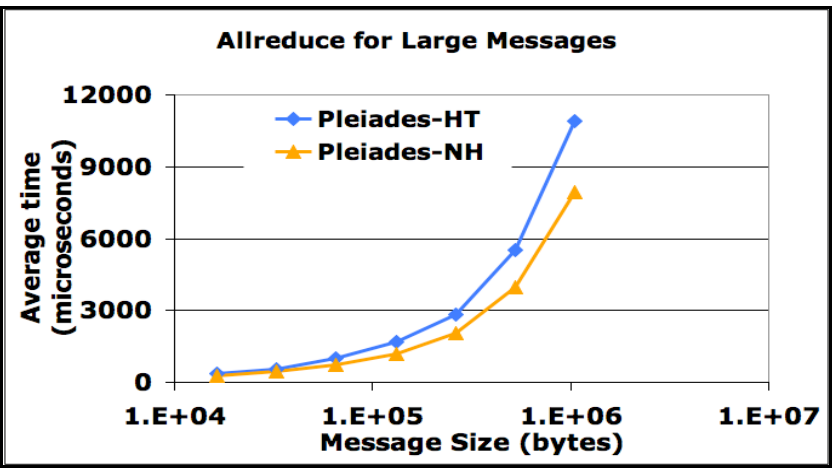

Figure 24: Performance of MPI_Allreduce on two systems for small messages.

Figure 25 shows the performance of MPI_Allreduce on two systems for a message size of 8 bytes used in MITgcmUV. On both systems up to 64 cores, performance of MPI_Allreduce is same and degrades slowly as the number of cores increase. It may be recalled that in MITgcmUV the average size of message broadcast is 8 bytes. Since the message size is very small the performance of MPI_Allreduce in MITgcmUV depends on the network latency of the system. Network latency of both systems increases with increasing number of cores especially beyond 128 cores ( 1 IRU) and therefore degrades rapidly.

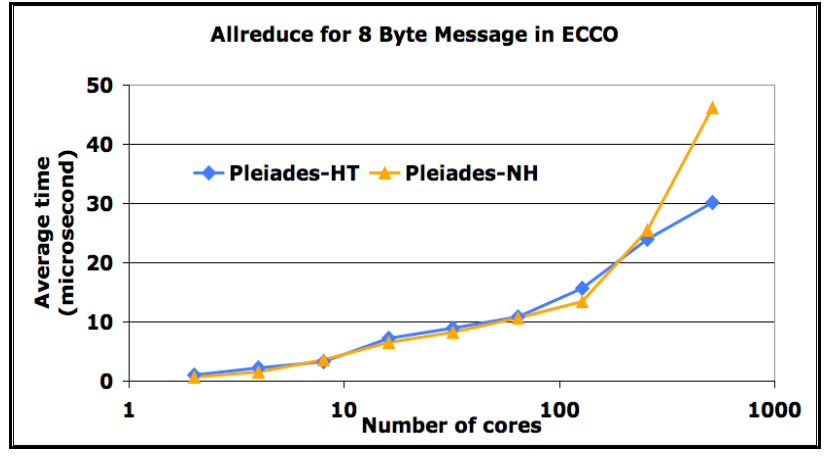

Figure 25: Performance of MPI_Allreduce on two systems for an 8-byte message.

\subsubsection{MPI_Gatherv}

Figure 26 shows the performance of MPI_Gatherv on two systems for small messages. Up to a message size $4 \mathrm{~KB}$, performance of Pleiades-HT is much better than Pleiades$\mathrm{NH}$, however for $8 \mathrm{~KB}$ message performance of Pleiades$\mathrm{NH}$ is better. The reason for this is the change in algorithm for the implementation of MPI_Gatherv on MPT.

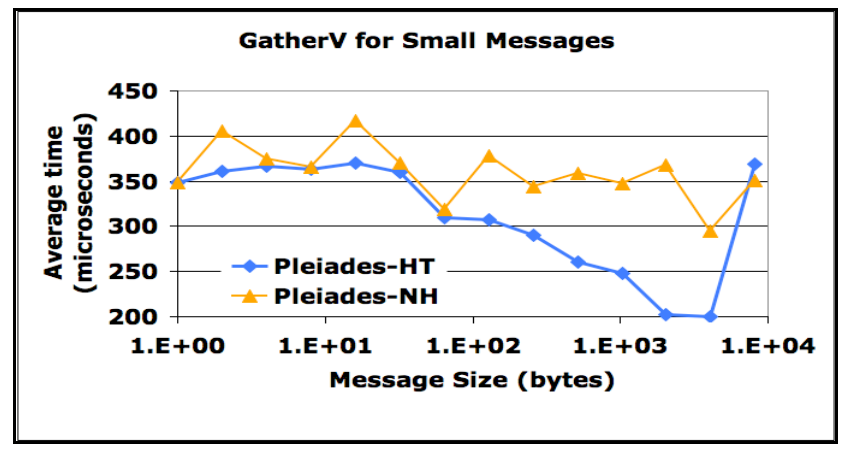

Figure 26: Performance of MPI_Gatherv on two systems for small messages.

Figure 27 shows the performance of MPI_Gatherv on two systems for large messages. Up to $64 \mathrm{~KB}$, performance of Pleiades-HT is better than Pleiades-NH, however for 128 $\mathrm{KB}$ to $1 \mathrm{MB}$ performance of Pleiades-NH is better.

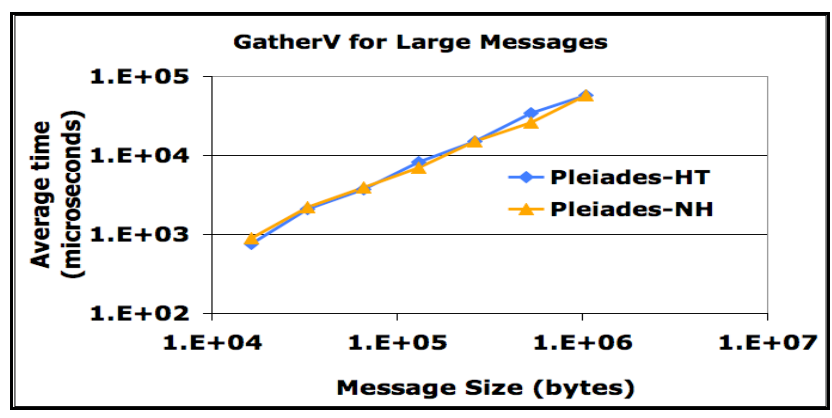

Figure 27: Performance of MPI_Gatherv on two systems for large messages.

Figure 28 shows the performance of MPI_Gatherv on two systems for a message $262 \mathrm{~KB}$. It is worth mentioning that 
average message in MPI_Gatherv is $270 \mathrm{~KB}$. Within a node ( 8 cores), performance of Pleiades-NH is better than Pleiades-HT - the former's inter-socket communication is faster due to QPI. Performance of both systems is same for 16 to 64 cores. Beyond 64 cores, performance of Pleiades$\mathrm{NH}$ is better than Pleiades-HT.

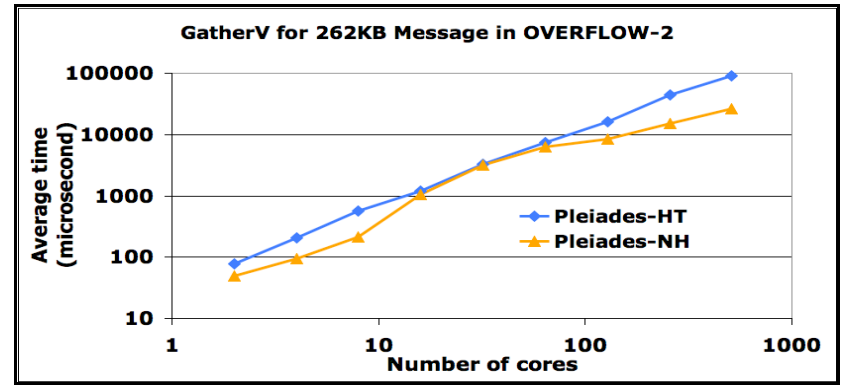

Figure 28: Performance of MPI_Gatherv on two systems for a $262 \mathrm{~KB}$ message.

\section{Summary and Conclusions}

In this paper, we study the performance of two NASA applications using two different analysis tools, TAU from University of Oregon and SGI's MPInside. We focus particularly on the communication times analyzing the performance of various MPI functions used in these applications. One of the most interesting results reached by our analysis is that relatively few functions in the MPI library are used in the MITgcmUV and OVERFLOW applications. The other conclusion is that write data (solution file) is relatively small, namely $2 \mathrm{~GB}$ and $8 \mathrm{~GB}$ for OVERFLOW and MITgcmUV, respectively, and is performed sequentially.

There was wide variation in message lengths - the shortest is 8-byte messages in MPI_Allreduce in MITgcmUV, and the largest message length is $1.3 \mathrm{MB}$ for MPI Bcast in OVERFLOW. Message length for MPI_Gatherv and MPI_Recv used in OVERFLOW is 270 bytes and $100 \mathrm{~KB}$, respectively. Average message length for $M P I$ Recv and MPI_Bcast used in MITgcmUV is $6 \mathrm{~KB}$ (actually 3 to 9 $\mathrm{KB}$ ) and $225 \mathrm{~KB}$. Overall, the conclusion that can be drawn is that inter-core communication for hardware and software must be optimized for both short and long messages. This paper shows that a large percentage of messages, for these applications, are not extremely long.

We used two different tools for analyzing the performance of the MPI benchmarks and the two applications: SGI's MPInside and TAU from University of Oregon. TAU has more extensive, sophisticated features and a nice visual interface. However, it does have a steep learning curve and to use it effectively, it is helpful to have support and training. On the other hand, MPInside is easy to use for the basic MPI functions but needs experience and training for collectives. Also, MPInside needs a better user interface and more features such as support to calculate the average message sizes.

\section{REFERENCES}

[1] Intel 5400 Chipset - Technical Documents, www.intel.com/Products/Server/Chipsets/5400/5400technicaldocuments.htm

[2] SGI Altix ICE, http://www.sgi.com/products/servers/altix/ice/configs. $\underline{\mathrm{html}}$

[3] Intel Microarchitecture (Nehalem), $\mathrm{http} / /$ www.intel.com/technology/architecturesilicon/next-gen/

[4] "An Introduction to the Intel ${ }^{\circledR}$ QuickPath Interconnect," Document Number: 320412, January 2009.

[5] InfiniBand Trade Assoc., www.infinibandta.org/home

[6] Message Passing Toolkit (MPT) User's Guide, http://techpubs.sgi.com/library/manuals/3000/0073773-003/pdf/007-3773-003.pdf

[7] HPCToolkit, http://www.hipersoft.rice.edu/hpctoolkit/

[8] IMP, http://ipm-hpc.sourceforge.net/

[9] KOJAK, http://icl.cs.utk.edu/kojak/

[10] mpiP, http://sourceforge.net/projects/mpip

[11] PAPI, http://icl.cs.utk.edu/papi/

[12] PDT (Program Database Toolkit), http://www.cs.uoregon.edu/research/pdt

[13] SvPablo, http://www.renci.org/projects/pablo.php

[14] TAU Performance System ${ }^{\circledR}$, http://tau.uoregon.edu

[15] MPInside Reference Manual 3.01, SGI, 2010.

[16] Scalasca (Scalable Performance Analysis of LargeScale Applications), http://www.fzjuelich.de/jsc/scalasca/

[17] OpenSpeedShop: http://techpubs.sgi.com/library/tpl/cgibin/browse.cgi? coll $=0650 \& \mathrm{db}=\mathrm{bks} \& \mathrm{~cm} d=$ toc $\& \mathrm{pth}=/ \mathrm{S}$ GI Developer/SShop UG

[18] Vampir - Performance Optimization, http://www.vampir.eu

[19] Paraver, http://www.bsc.es/plantillaA.php?cat id=485

[20] Jumpshot, http://wwwunix.mcs.anl.gov/perfvis/software/viewers/

[21] Vtune, http://www.intel.com/software/products/vtune/vlin/index.ht $\underline{m}$

[22] PerfSuite, http://perfsuite.ncsa.uiuc.edu/

[23] OVERFLOW-2, http://aaac.larc.nasa.gov/ buning/

[24] ECCO: Estimating the Circulation and Climate of the Ocean, www.ecco-group.org/

[25] Intel MPI Benchmarks: Users Guide and Methodology Description, Intel GmbH, Germany. 